\title{
Inadequate corticosterone levels relative to arthritic inflammation are accompanied by altered mitochondria/cholesterol breakdown in adrenal cortex: a steroid-inhibiting role of IL-1 $\beta$ in rats
}

\author{
Christine Wolff, ${ }^{1}$ Katharina Krinner, ${ }_{1}^{1}$ Josef A Schroeder, ${ }^{2}$ Rainer H Straub ${ }^{1}$
}

\begin{abstract}
Handling editor Tore K Kvien
- Additional material is published online only. To view please visit the journal online (http://dx.doi.org/10.1136/ annrheumdis-2013-203885)

${ }^{1}$ Laboratory of Experimental Rheumatology and Neuroendocrino-Immunology, Division of Rheumatology, Department of Internal Medicine I, University Hospital Regensburg, Regensburg, Bavaria, Germany

${ }^{2}$ Department of Pathology, University Hospital Regensburg, Regensburg, Bavaria, Germany
\end{abstract}

Correspondence to Professor Rainer H Straub, Laboratory of Experimental Rheumatology and Neuroendocrino-Immunology, Division of Rheumatology, Department of Internal Medicine I, University Hospital, Regensburg 93042, Germany; rainer.straub@klinik.uniregensburg.de

Received 4 May 2013 Revised 12 April 2014 Accepted 16 April 2014 Published Online First 8 May 2014

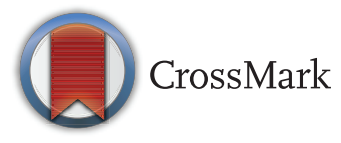

\footnotetext{
To cite: Wolff $C$, Krinner $K$, Schroeder JA, et al. Ann Rheum Dis 2015;74: 1890-1897.
}

\section{ABSTRACT}

Objectives In rheumatoid arthritis, inadequate cortisol secretion was observed relative to inflammation, but reasons are unknown. Human adrenal glands cannot be investigated due to ethical reasons. Thus, a model of arthritis was studied to test inadequate glucocorticoid secretion and adrenocortical alterations.

Methods Arthritis in DA rats was induced by collagen type II. Plasma hormone (cytokine) levels were determined by ELISA or radioimmunoassay (Luminex). Adrenocortical cells were investigated making use of in vitro culture, immunohistochemistry and imaging techniques, cholesterol uptake studies and electron microscopical morphological analyses of adrenocortical lipid droplets and mitochondria.

Results During the course of arthritis, corticosterone and adrenocorticotropic hormone (ACTH) levels were only elevated on day 1 after immunisation but were in the normal range from day 5 to 55 . Serum levels of corticosterone relative to IL-1 $\beta$ were markedly lower in arthritis than in controls. IL-1 $\beta$ inhibited ACTH-stimulated corticosterone secretion from adrenocortical cells in vitro. Cholesterol uptake receptor SR-BI protein was unchanged. Number of altered swollen and cavitated mitochondria increased during the course of arthritis (maximum on day 55), and this was correlated to reduced breakdown of lipid droplets and increased Sudan III-positive lipid accumulation from day 28 to 55 . Reduced lipid breakdown measured as a high number of homogenous lipid droplets negatively correlated with plasma corticosterone $(p=0.022)$. Adrenocortical tissue density of normal mitochondria positively correlated with serum corticosterone levels.

Conclusions This study on inadequate adrenal glucocorticoid secretion in arthritis demonstrated altered mitochondria and altered lipid breakdown paralleled by low corticosterone levels in relation to inflammation. $\mathrm{IL}-1 \beta$ is a key cytokine.

\section{INTRODUCTION}

Rheumatologists use glucocorticoids in the therapy of chronic inflammatory diseases, and glucocorticoids have strong anti-inflammatory and antiproliferative effects. ${ }^{1} 2$ Early studies reported that decreased adrenocortical function exists in patients with chronic inflammatory diseases such as rheumatoid arthritis (RA). ${ }^{3}$ Similar concepts evolved in critically ill patients who can develop long-standing relative adrenal insufficiency. ${ }^{4}{ }_{5}^{5}$ The initial disposition to believe in the presence of low hypothalamic-pituitary-adrenal (HPA) axis activity (1950-late 1990s) has been qualified in human chronic inflammatory diseases. $^{6}$

One of the most striking findings was described as relatively low levels of glucocorticoids in relation to inflammation. ${ }^{7}$ This can be directly studied using a hormone-to-cytokine ratio of cortisol and IL-6 in humans, which demonstrates low levels of cortisol relative to IL-6 in patients with RA and reactive arthritis as compared to healthy controls. ${ }^{8}$ In addition, experimental arthritis in DA rats was accompanied by relatively low levels of corticosterone relative to inflammatory conditions. ${ }^{9}$ However, the reasons for this phenomenon in RA, reactive arthritis and experimental arthritis are still not known.

Several local factors determine generation of glucocorticoids in the adrenal glands: (1) cholesterol uptake via the high-density lipoprotein (HDL) cholesterol scavenger receptor class B member 1 (SR-B1); (2) vacuolar storage and breakdown of cholesterol via lipases such as the hormonesensitive lipase and the adipose triglyceride lipase; (3) stimulatory factors such as adrenocorticotropic hormone $(\mathrm{ACTH})$, but also cytokines like IL-1 IL-6, TNF or neuropeptides that determine uptake and steroid conversion; (4) steroid-converting enzymes and their multiple cofactors that can be inhibited by TNF and IL-1 $\beta$; (5) mitochondrial function because several steroid conversion steps happen within mitochondria; and (6) secretion of glucocorticoids. To our knowledge, of these different factors, none has been investigated in experimental arthritis.

In particular, the investigation of mitochondria can be important because these organelles serve steroidogenesis and ATP production in cells of the adrenal cortex. Mitochondria can undergo marked ultrastructural changes upon inhibition of important enzyme steps of steroidogenesis. This has been demonstrated using aminoglutethimide, a blocker of the side-chain cleavage enzyme P450scc that converts cholesterol to pregnenolone, a precursor of glucocorticoids. ${ }^{10}$ Since inflammatory cytokines such as IL-1 $\beta$ and TNF can similarly interfere with these important steps of steroidogenesis (reviewed in ref. 11), one might expect ultrastructural changes of adrenocortical mitochondria during the course of inflammatory illness. 
In experimental arthritis, we hypothesise that we can observe (A) inadequate corticosterone secretion in relation to inflammation, (B) a cytokine responsible for inhibition of corticosterone secretion, (C) a loss of surface expression of SR-B1 and changes of cholesterol storage/droplets in adrenocortical cells, and (D) abnormal mitochondria in the course of the disease. This study included respective parameters to delineate possible adrenocortical defects in experimental arthritis.

\section{MATERIALS AND METHODS}

Animal experiments were conducted according to institutional and governmental regulations for animal use (Government of the Oberpfalz AZ 54-2532.1-06/09). Species and vendors are described in the online supplementary material. Collagen type II-induced arthritis was induced and scored with standard techniques as given in the online supplementary material. Corticosterone, ACTH and cytokines (IL-1 $\beta$ and IFN $\gamma$ ) were measured by radioimmunoassay and ELISA techniques (see online supplementary material). In vitro superfusion methods are described in the online supplementary text.

Quantitative analysis of adrenocortical surface SR-B1 expression was done with the help of immunohistochemistry and image analysis. Sudan III staining, light microscopy and image analysis were used to quantify cholesterol storage in adrenocortical cells. Cholesterol uptake into adrenocortical cells was studied with fluorescent NBD-cholesterol and subsequent FACS analysis (all techniques described in the online supplementary material).

Ultrastructural analysis of adrenocortical lipid droplets and mitochondria was carried out with standard energy-filtered transmission electron microscopy and image analysis as described in the online supplementary material. Thereby two types of lipid droplets were distinguished: (1) smooth lipid droplets that are completely homogenous, and (2) inhomogenous lipid droplets with vacuolar bodies. Furthermore, electron micrographs $(3150 \times)$ were evaluated concerning mitochondria morphology. To study stimulus-induced changes of lipid morphology, adrenocortical cells were stimulated with either ACTH or the $\beta$-adrenergic agonist isoproterenol, and lipid morphology was characterised as mentioned in the online supplementary material.

Statistical analyses were performed by standard techniques, and a p value below 0.05 was the significance level (see online supplementary material).

\section{RESULTS}

\section{Inflammation increases adrenal and spleen weight}

In order to estimate clinically relevant inflammation, we studied clinical arthritis score and body weight. Arthritis started with clinical symptoms on days 14-16 (maximum around days 24-26 after immunisation) (figure $1 \mathrm{~A}$ ). At day 14, body weight dropped, which continued until days 24-26 (figure 1B). After days 24-26, despite maximal arthritis scores, body weight increased at a similar rate compared with controls (figure 1B).

Importantly, weight of the adrenal glands already increased on day 1 after immunisation, but declined to near normal levels on day 5 , indicating an acute response (figure 1C). However, at maximal clonal expansion of autoreactive lymphocytes expected on day 14 (figure 1C), adrenal weights were high again, and this remained stable until day 41 (figure 1C). In the late chronic phase of the disease, adrenal gland weights became again normal on day 55 (figure $1 \mathrm{C}$ ), which in the light of ongoing inflammation might be a sign of inadequacy.
In contrast, spleen weights increased until day 14 and then remained stable throughout the entire observation period indicating a continuous immune response (figure 1D).

\section{Inadequate secretion of HPA axis hormones in relation to inflammation}

Parallel to increased adrenal gland weights on day 1 , we observed a marked increase of corticosterone on day 1 (figure $2 \mathrm{~A}$ ). However, this normalised on day 5 , and despite elevated weights of adrenal glands between days 14 and 41 and a steadily increasing IL-1 $\beta$, corticosterone remained normal (figure 2A). This remarkable finding was similar for plasma levels of ACTH (figure 2B).

To directly study inadequacy, IL-1 $\beta$, IL-6, TNF and IFN- $\gamma$ were measured in plasma in order to generate a hormone/cytokine ratio. Of these cytokines, only IL- $1 \beta$ demonstrated a continuous increase over time during the entire course of arthritis (figure 2C). IFN- $\gamma$ increased until day 5 and subsequently decreased to normal levels (see online supplementary figure S1a), which was similar for IL-6 (data not shown). TNF was not measurable. Thus, IFN- $\gamma$ and IL- 6 are not suitable to demonstrate inadequacy throughout observation time.

Now, we generated a ratio of plasma corticosterone divided by plasma IL-1 $\beta$. Figure $2 \mathrm{D}$ shows that this particular ratio was higher in control animal compared with arthritic animals. A similar ratio of plasma corticosterone and IFN- $\gamma$ was generated, but due to the low levels in the overt phase of arthritis, this ratio was not suitable to demonstrate inadequacy (see online supplementary figure S1b).

Usually, secretion of ACTH is followed by secretion of glucocorticoids with a time interval of $15-30 \mathrm{~min}$ as known from the corticotropin-releasing hormone test. ${ }^{12}$ Thus, one expects that there exists a positive correlation between plasma ACTH and serum glucocorticoid levels. Such a positive correlation existed for plasma ACTH and serum corticosterone in control animals (see online supplementary figure S1c) and also for immunised rats on day 1 , the day of maximum corticosterone surge (see online supplementary figure S1d). This indicates that ACTH and corticosterone are coupled in their secretion patterns under healthy and very early inflammatory conditions. However, in later phases of arthritis, no such positive interrelation existed (uncoupling, see online supplementary figure S1e).

\section{IL-1ß inhibits ACTH-stimulated secretion of corticosterone}

Since IL-1 $\beta$ was a good marker to demonstrate inadequacy, we tested whether IL-1 $\beta$ can influence ACTH-stimulated corticosterone secretion from adrenal cells and tissue. Under control conditions without inflammation, ACTH stimulated corticosterone secretion from adrenocortical tissue slices in superfusion chambers (figure 2E) and adrenal tissue in culture wells (figure 2F). This stimulatory influence of ACTH was also observed in day $1 \mathrm{immu-}$ nised animals (figure 2E), but ACTH was not able to stimulate corticosterone secretion in late arthritic animals (figure 2E). Under control conditions (figure 2E,F) and on day 1 after immunisation (figure $2 \mathrm{E}$ ), IL-1 $\beta$ inhibited ACTH-stimulated corticosterone secretion. This clearly indicates that IL-1 $\beta$ is a critical inhibitory molecule that is most probably responsible for inadequacy in DA rats.

\section{Cholesterol uptake and lipid storage in adrenocortical cells}

The precursor of glucocorticoid generation is cholesterol ester that is taken up by adrenocortical cells using the HDL cholesterol receptor SR-B1. ${ }^{13}$ SR-B1 protein expression did not differ in controls compared with arthritic animals throughout the observation phase (figure 3A). 
A

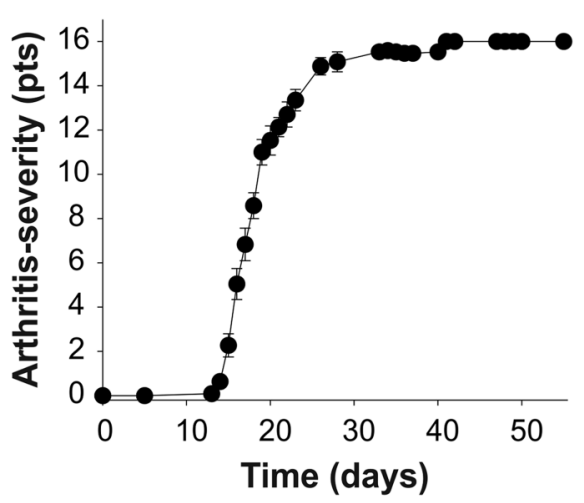

B $\quad \square \quad \begin{aligned} & \text { control animals } \\ & \text { immunized animals }\end{aligned}$

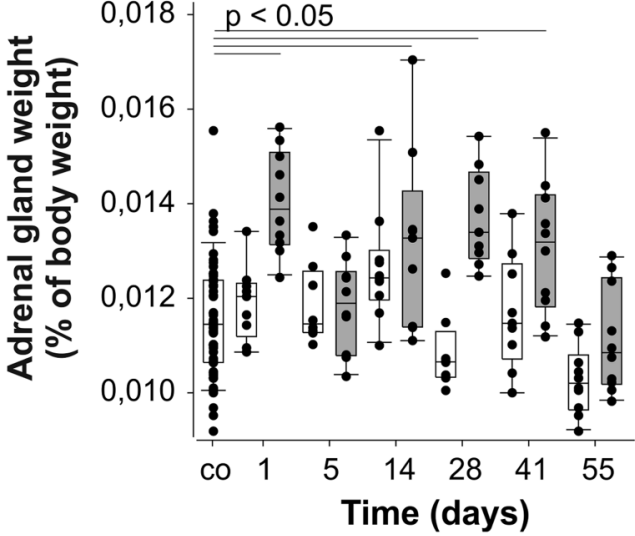

B $\quad-0-$ control animals
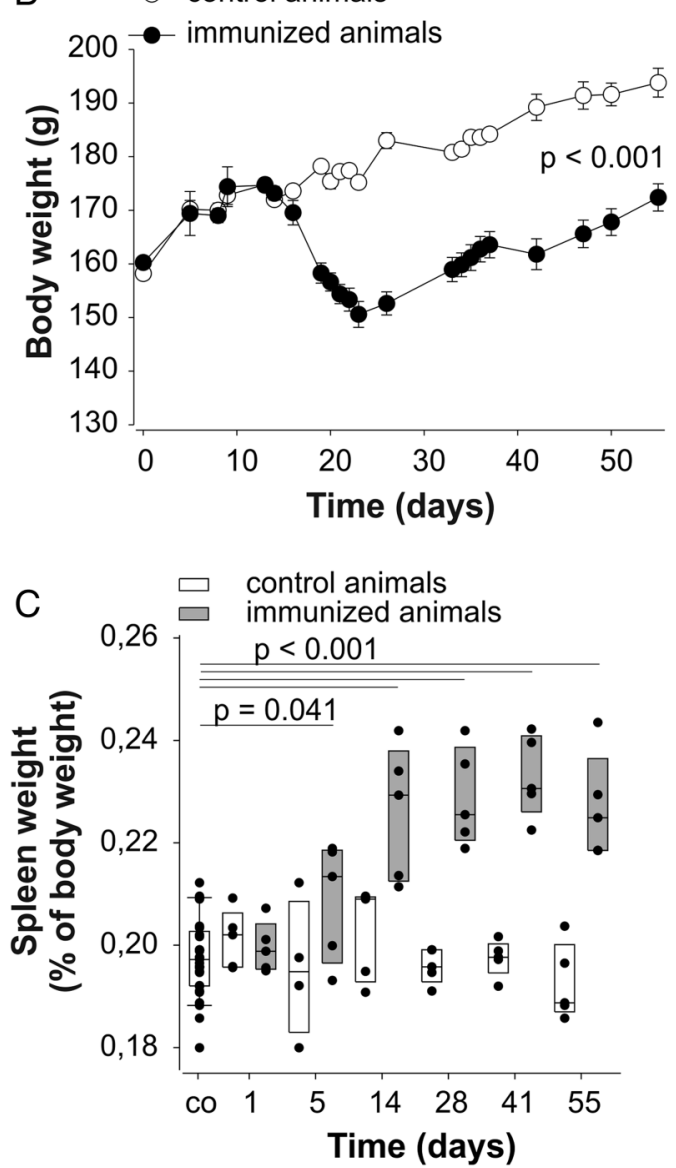

Figure 1 Course of clinical arthritis score (A), body weight (B), weight of adrenal glands (C) and spleen weight (D) in experimental arthritis. In all experiments, at least 6-12 rats were studied at each time point. In panels (A) and (B), data are given as median with 25/75 IQR. The p-value in panel (B) reflects the outcome of a general linear model analysis that compared both experimental groups over all time points. In panels (C) and (D), the number of rats is given by circular symbols, which sometimes superimpose on each other. The mean value of adrenal weights in controls amounted to $15.4 \mathrm{mg}$ and of spleens $359 \mathrm{mg}$. co, control animals (without arthritis). Data in box plots represent the 25th to 75th centiles, the lines within the boxes represent the median and the lines outside the boxes represent the 10th and 90th centiles.

We next focused on lipid storage in adrenocortical cells. Lipid storage was significantly increased between controls and arthritic animals on day 28 (figure 3B). The lipid storage was also increased on days 28-55 (combined data) compared with days 5 and 14 (combined data) (figure 3B). In addition, control values (13.4\% (9.2-17.1)) were not different from combined data of days 5 and $14(\mathrm{p}=0.391)$ but were lower compared with combined data of days 28 and $55(\mathrm{p}=0.017)$. This indicates that from day 28 onwards, despite ongoing inflammation, adrenal lipid storage was higher in experimental arthritis than in controls.

In order to study cholesterol uptake into adrenocortical cells, the NBD-cholesterol uptake test was applied. NBD cholesterol uptake was not different in control animals compared with immunised/arthritic animals throughout the entire observation period (figure 3C). In addition, there existed a positive interrelation between NBD cholesterol uptake and serum levels of corticosterone in control animals but not in immunised/arthritic animals (figure 3D), which might be interpreted as uncoupling of precursor uptake and secreted glucocorticoids.

\section{Characterisation of lipid droplets in adrenocortical cells and influence of inflammation}

Storage of cholesterol in adrenocortical cells happens in lipid droplets. One can observe two types of lipid droplets, one with smooth homogenous and one with inhomogenous vacuolar content (figure 4A). In order to learn why some droplets are inhomogenous and others homogenous, we investigated these droplets in wild-type mice and adipose triglyceride lipase (ATGL)-deficient mice. ${ }^{15}$ Most lipid droplets were inhomogenous in wild-type animals, while ATGL-deficient mice do not possess inhomogenous droplets (figure 4B). This might indicate that inhomogenous lipid droplets represent activated lipid droplets that undergo lipid breakdown, and ATGL-deficient animals cannot breakdown these lipid droplets.

To further characterise morphology of lipid droplets, adrenocortical cells isolated from adrenal glands of DA rats were stimulated with ACTH or isoproterenol, which resulted in increased numbers of inhomogenous lipid droplets and smaller numbers of homogenous lipid droplets (figure 4C).

In addition, there was a negative correlation between density of inhomogenous and homogenous lipid droplets in control and immunised animals with similar correlation coefficients (figure 4D). This physiological part of our studies helped to characterise two types of lipid droplets, and it also demonstrates that more inhomogenous lipid droplets are accompanied by less homogenous lipid droplets and vice versa.

Finally, the number of inhomogenous (homogenous) lipid droplets was higher (lower) in control animals compared with 

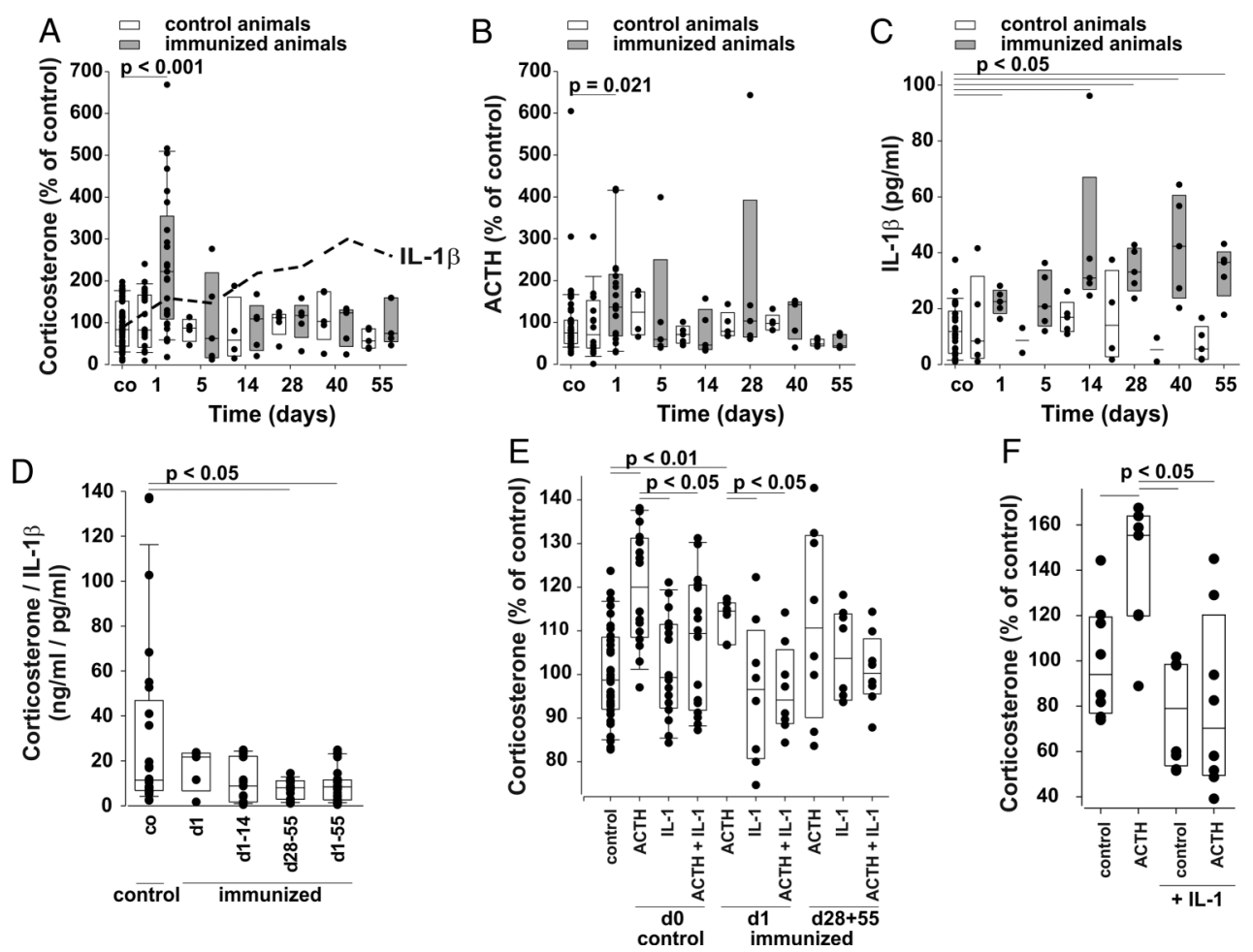

Figure 2 Inadequate secretion of hypothalamic-pituitary-adrenal axis hormones in experimental arthritis. The number of animals in these experiments is indicated by a circular symbol in the panels (at least $n=6)$. (A) Course of serum corticosterone in controls (co, $100 \%=238 \mathrm{ng} / \mathrm{mL}$ ) and immunised/arthritic animals. (B) Course of plasma adrenocorticotropic hormone (ACTH) in controls $(100 \%=12.1 \mathrm{pg} / \mathrm{mL})$ and immunised/arthritic animals. (C) Plasma IL-1 $\beta$ in controls (co) and immunised/arthritic rats throughout observation time. (D) Ratio of serum corticosterone divided by serum IL-1 $1 \beta$ in controls and immunised/ arthritic animals. (E) Influence of IL-1 $\beta$ on ACTH-stimulated corticosterone secretion. Adrenal tissue slices of control rats (day 0 ) and immunised/arthritic rats on day 1, day 28 and day 55 were superfused in the superfusion microchambers in vitro. (F) Influence of IL-1 $\beta$ on ACTH-stimulated corticosterone secretion from cultured adrenocortical tissue of normal rats in vitro. Box plots are explained in legend to figure 1.

immunised animals, which surprisingly indicates reduced lipid droplet breakdown in immunised animals compared with control animals (figure 4E).

\section{Morphology of mitochondria in adrenocortical cells}

After breakdown of lipid droplets, released cholesterol is taken up as cholesteryl ester by mitochondria, which depends on complex transport mechanisms. ${ }^{16}$ In mitochondria, steroidogenesis is started by the P450scc side chain cleavage enzyme that converts cholesterol into pregnenolone.

In our studies, we observed normal and altered mitochondria (figure 5A). Normal mitochondria demonstrated homogenous patterns of vesicular structures evenly distributed throughout the cross section. Altered mitochondria were characterised by swelling, rarefication of cristae/tubuli, more dispersed vesicles in the matrix, and cavitations within the matrix (figure 5A). In control animals, the density of altered mitochondria was six times lower as compared with the density of normal mitochondria throughout 55 days (figure 5B). However, the density of normal mitochondria markedly decreased in immunised/arthritic animals while the density of altered mitochondria significantly increased from day 0 to 55 (figure $5 \mathrm{~B}$ ). This indicates marked alterations in mitochondria morphology in experimental arthritis.

\section{Related functional consequences of altered mitochondria and type of lipid droplets}

The density of normal mitochondria positively correlated with the density of inhomogenous lipid droplets in all animals and in the subgroup of control animals (figure 6A). In contrast, the density of altered mitochondria negatively correlated with the density of inhomogenous lipid droplets in all animals, and in the two subgroups of control animals and immunised/arthritic animals (figure 6B). This indicates that the presence of normal mitochondria is related to increased lipid droplet breakdown while the presence of altered mitochondria is linked to decreased lipid droplet breakdown.

Although many different factors can influence serum corticosterone levels such as degradation in tissue such as the liver, renal elimination and binding to transport proteins, we hypothesised that mitochondria morphology might translate into different levels of serum corticosterone. The density of normal mitochondria was positively correlated to serum corticosterone levels when using data of all animals (figure 6C). Due to the lower number of observations (type II error), this interrelation was not present in the individual subgroups (figure 6C). In addition, there was no significant interrelation between density of altered mitochondria and serum corticosterone levels, but the estimated regression line had a negative slope (figure 6D).

Finally, the number of homogenous lipid droplets and corticosterone levels was negatively correlated in immunised/arthritic animals but not in controls (see online supplementary figure S2a,b). In addition, a trend towards a positive correlation was detected between the number of inhomogenous lipid droplets and corticosterone levels in immunised/arthritic animals but not in controls (see online supplementary figure S2c,d).

\section{DISCUSSION}

This study demonstrated inadequate HPA axis hormone levels relative to systemic inflammation as measured by serum levels of IL-1 $\beta$, IL-1 $\beta$-induced inhibition of ACTH-stimulated 

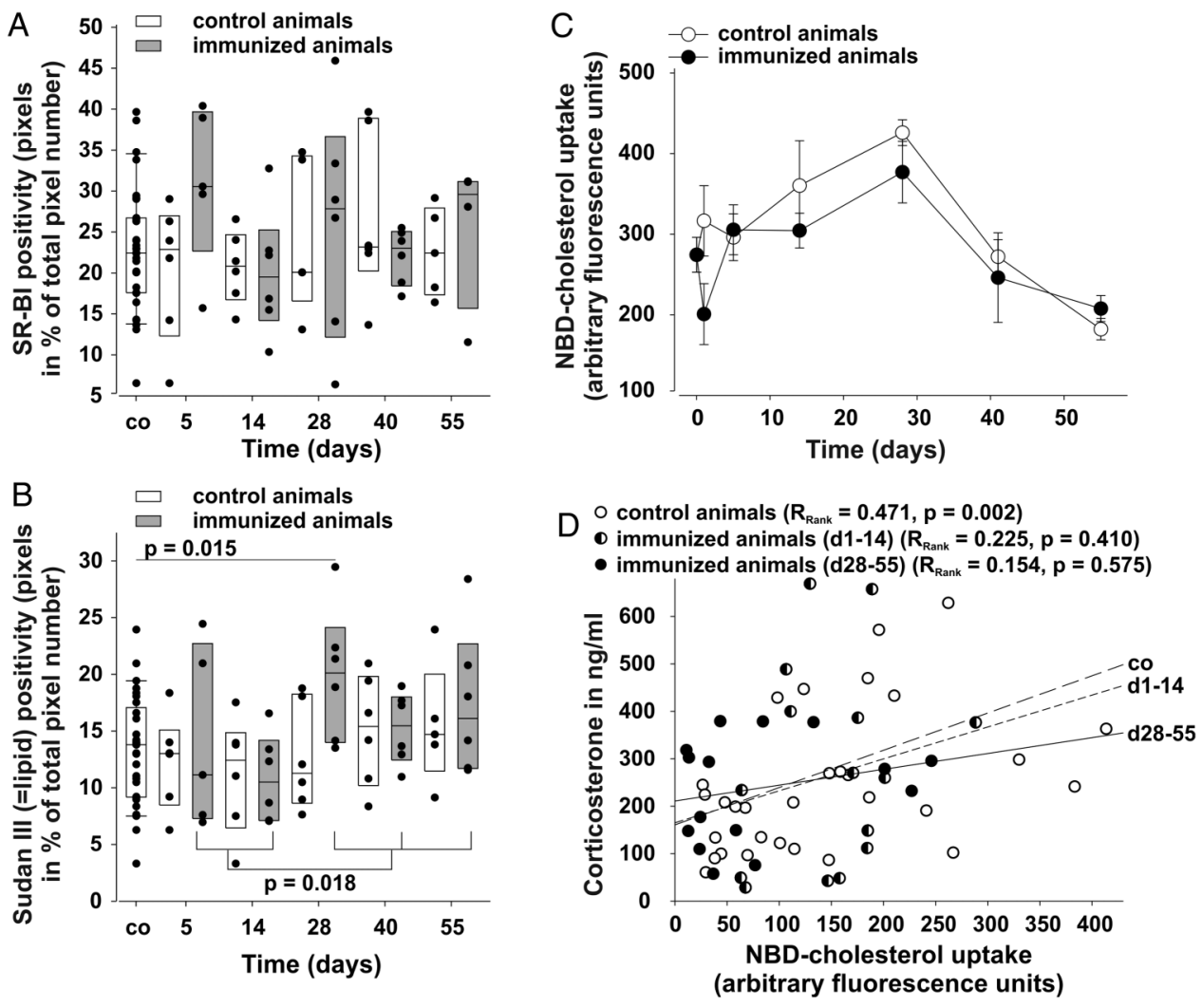

Figure 3 Cholesterol provision to and uptake in adrenocortical cells during experimental arthritis. The number of animals in these experiments is indicated by a circular symbol in the panels (at least $n=6$ ). Box plots are explained in legend to figure 1. (A) Expression of the high-density lipoprotein cholesterol receptor SR-B1 over time. (B) Sudan III positivity (lipid positivity) of adrenocortical cells over time. The p-value stands for the comparison of all data at days 5/14 with days 28/40/55 in immunised animals (only grey boxes). (C) NBD cholesterol uptake to adrenocortical cells during the observation period of 55 days. Data are given as median with 25/75 IQR. (D) Interrelation of NBD cholesterol uptake and serum corticosterone levels. The linear regression lines and Spearman rank correlation coefficients $\left(R_{\text {Rank }}\right)$ are given in the panel.

corticosterone secretion from adrenal glands, normal SR-B1 expression throughout illness, increased lipid depots in the later phase of arthritis compared to control and preclinical phases, a higher number of altered cavitated mitochondria in experimental arthritis than in controls and a positive interrelation between density of normal mitochondria and lipid droplet activation as well as serum corticosterone.

Relatively inadequate secretion of glucocorticoids but also ACTH in systemic inflammation is a hallmark of chronic inflammatory diseases in humans, ${ }^{6} 7{ }^{17-19}$ but also in animals with arthritis. $^{9} 20$ This inadequacy applies not only to patients with chronic inflammatory diseases but also to other inflammatory conditions. For example, a blunted HPA axis response to repeated IFN- $\alpha$ injections was demonstrated in patients with myeloproliferative disease over 3 weeks. ${ }^{21}$ The effect is really impressive because ACTH and cortisol responses were nearly blunted. ${ }^{21}$ In this present study, we were able to confirm relatively low levels of glucocorticoids relative to inflammation in experimental arthritis despite increased weights of adrenal glands. Increased weights of adrenal glands have been described earlier in arthritis, ${ }^{20} 22$ demonstrating a very similar time course in rats. ${ }^{20}$

In this study, IL-1 $\beta$ was the marker of inflammation because it was perfectly measurable in rats. While in humans often IL-6 is used as a maker to demonstrate inadequate secretion of glucocorticoids in relation to inflammation, the measurable IL-6 is only a downstream readout parameter of prior IL-1 $\beta$ and TNF secretion (IL-1 $\beta$ and TNF can hardly be measured in human serum). However, IL-1 $\beta$ and TNF are most probably the critical elements that interfere with adrenal steroidogenesis as recently summarised. ${ }^{11}$ Fortunately, IL-1 $\beta$ can be measured in rats and, thus, the ratio of corticosterone/IL- $1 \beta$ is more appropriate to demonstrate inadequacy than the ratio of cortisol/IL-6. In this study in rats, neither ratio of corticosterone/IL-6 nor corticosterone/IFN- $\gamma$ were applicable because both cytokines were not continuously high and, thus, do not represent a suitable inflammation marker.

In earlier theoretical work, we hypothesised that a short rise and fall of ACTH/cortisol was evolutionarily positively selected in order to support an early response of but not a long-term inhibitory influence on the immune system. ${ }^{23}$ Such an adaptive programme would support the described inadequacy. In this study, an expected immunisation-dependent corticosterone surge on day 1 in experimental arthritis should be downregulated, which was already observable on day 5. In controls and day 1 immunised animals, the positive correlation of corticosterone and ACTH levels demonstrates necessary coupling of the pituitary and adrenal glands, which gets lost in the later phases. The loss of the ACTH influence on adrenal glands during inflammation has recently been termed dissociation of ACTH and glucocorticoids. ${ }^{24}$ If this theory is correct, regulatory adaptive mechanisms should be in place that can downregulate HPA axis activation after an initial inflammatory stimulus.

Importantly, IL-1 $\beta$ - our marker of continuous inflammation in the rat-inhibited ACTH-stimulated corticosterone secretion from adrenocortical cells of normal rats but also from 

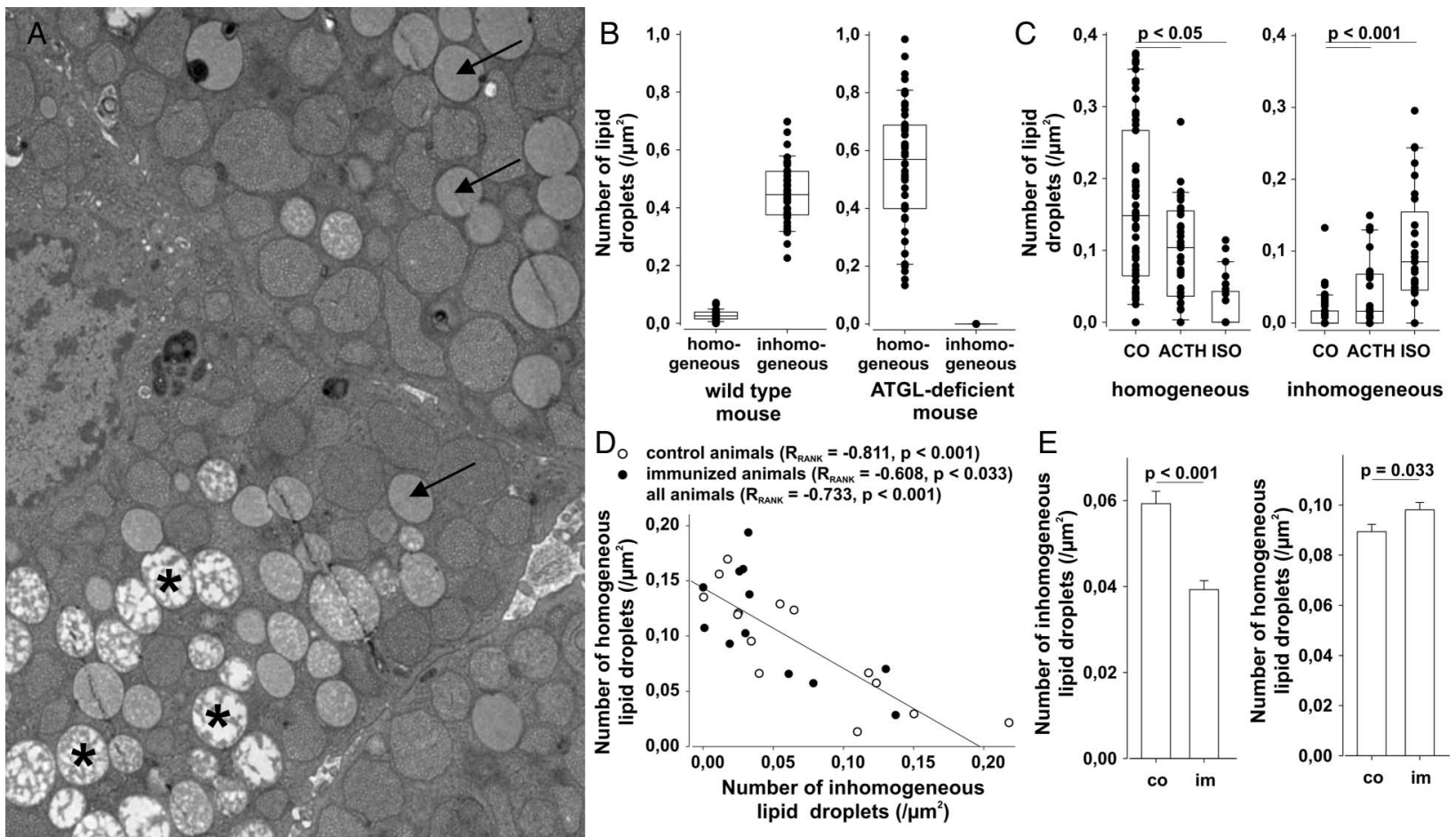

Figure 4 Characterisation of lipid morphology in the adrenal cortex. Box plots are explained in legend to figure 1. (A) Electron microscopy demonstrates homogenous (arrow) and inhomogenous (asterisk) lipid droplets. Magnification: 3150x. (B) Homogenous and inhomogenous lipid droplets in wild-type C57BL/6 mice and adipose triglyceride lipase (ATGL)-deficient C57BL/6 mice. ATGL-deficient mice do not possess inhomogenous lipid droplets. (C) Influence of adrenocorticotropic hormone (ACTH) and isoproterenol ( $\beta$-adrenergic agonist) on lipid droplets in adrenocortical cells from DA rats. Cells were either not stimulated $\left(C O=\right.$ control) or stimulated with ACTH $\left(\mathrm{ACTH}, 10^{-10} \mathrm{M}\right)$ or isoproterenol $($ ISO, $\left.10^{-6} \mathrm{M}\right)$. (D) Interrelation between number of inhomogenous and homogenous lipid droplets. The linear regression line of all animals and Spearman rank correlation coefficients of all, control and experimental animals $\left(R_{\text {Rank }}\right)$ are given. $(E)$ Number of (in-)homogenous lipid droplets in control (co) versus immunised/arthritic (im) animals. Data are given as means \pm SEM.

superfused adrenal tissue in vitro. In addition, ACTH cannot stimulate corticosterone secretion in late arthritic adrenal tissue in culture in vitro. Thus, IL- $1 \beta$ is a perfect candidate for the unfavourable influence on the adrenal glands. Similarly, IL-1 $\beta$ was now described as the critical proinflammatory factor in pancreatic $\beta$-cell destruction in humans with type 2 diabetes mellitus as recently demonstrated in randomised clinical trials with IL-1 receptor antagonist. ${ }^{25}$ One might speculate that the juvenile form of arthritis, in which IL-1 receptor antagonist works perfectly well, is more dependent on adrenal gland dysfunction than the adult form of RA.
Among other reasons ${ }^{16}$ the following important causes for HPA axis downregulation have been mentioned. (A) The initially elevated cortisol levels rapidly inhibit hypothalamic neurons stopping corticotropin-releasing hormone secretion (negative feedback), but cytokines such as IL-6 may take over adrenal glucocorticoid stimulation. ${ }^{24}$ (B) Uptake of HDL cholesterol to the adrenal gland might be downregulated. (C) Adrenal cholesterol stores might be emptied so that continuous production of glucocorticoids is impossible. While there is little doubt that mechanisms under item A apply for short-lived inflammation, aspects of item $\mathrm{B}$ or $\mathrm{C}$ have not been investigated in chronic
Figure 5 Changes in morphology of adrenocortical mitochondria during experimental arthritis. (A) Electron microscopy demonstrates normal (arrow) and altered (asterisk) mitochondria. Magnification: 3150x.

(B) Course of mitochondrial morphology in normal (circular symbols) and immunised/arthritic animals (triangular symbols).

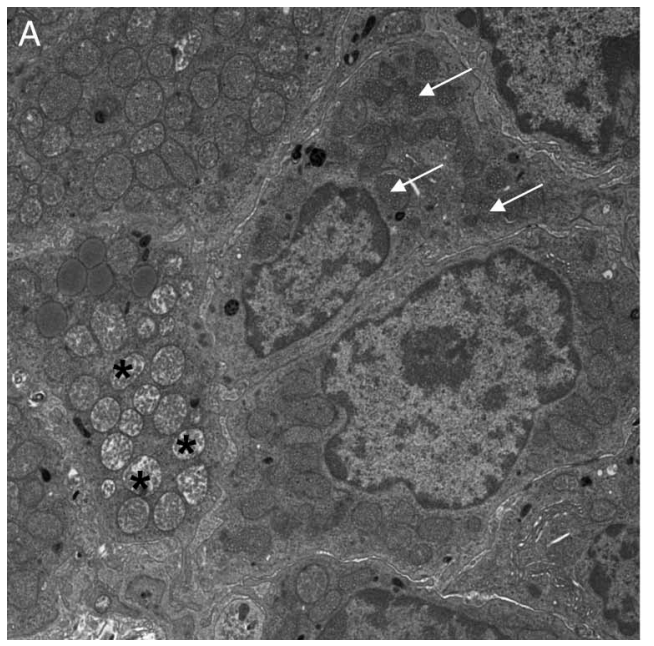

B - - normal mitochondria - control animals - normal mitochondria - immunized animal - altered mitochondria - control animals $\triangle-$ altered mitochondria - immunized animals

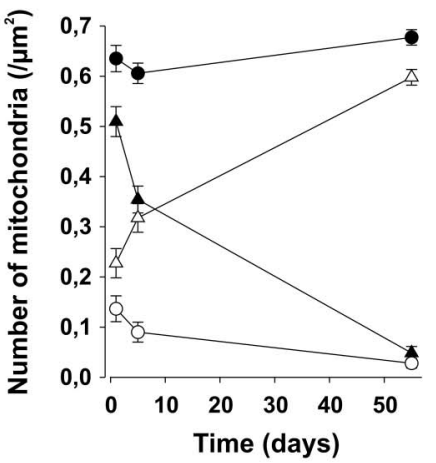



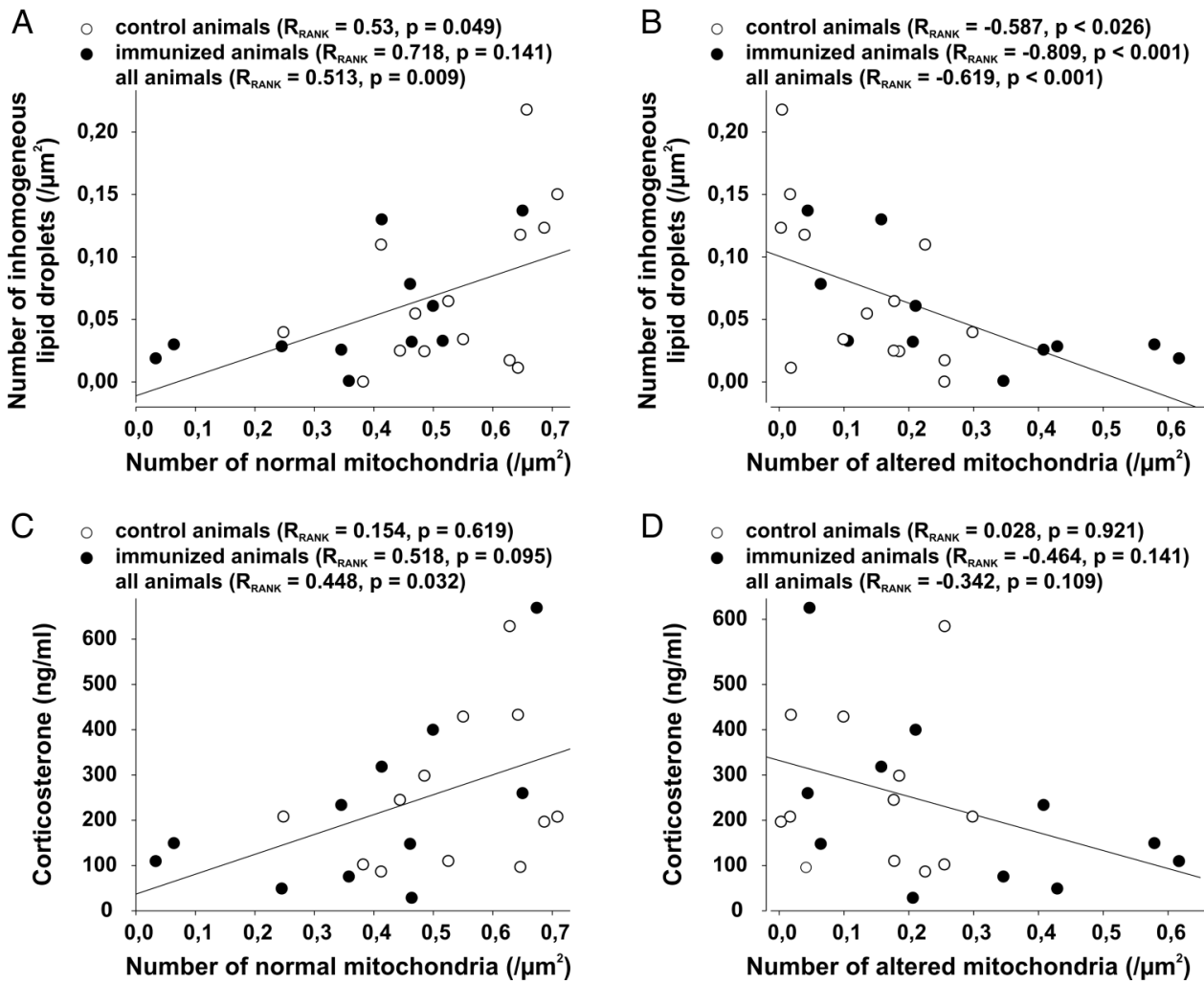

Figure 6 Possible functional consequence of altered mitochondria. In all panels, the linear regression line of all animals and Spearman rank correlation coefficients $\left(R_{\text {Rank }}\right)$ of all groups are given. $(A)$ and $(B)$ Interrelation of density of normal $(A)$ and altered (B) mitochondria and density of inhomogenous lipid droplets. (C) and (D) Interrelation between density of normal (C) and altered (D) mitochondria and serum levels of corticosterone. In all panels, data of animals at different time points during observation were included.

inflammatory arthritis. It turned out in this study that neither protein expression of the HDL cholesterol receptor SR-B1 nor cholesterol uptake were disturbed in experimental arthritis. In addition, there was no exaggerated emptying of lipid stores as objectified by Sudan III staining. Unexpectedly, lipid stores were even increased in late phase experimental animals as compared with controls and immunised pre-arthritic animals.

In a recent refinement of the above-given theory, we expounded that adaptive responses of the body are not positively selected for a long-lasting inflammatory illness. ${ }^{26} 27$ According to these theoretical considerations, the chronic inflammatory process starts between around week $6 .{ }^{27}$ From this point of view, adaptive responses might be expected until week 6 but not thereafter. Beyond this critical time point, long-standing immune responses and inflammation might lead to unwanted bystander alterations of the neuroendocrine and other systems. ${ }^{26}$ For example, prolonged increase of inflammatory stimuli with elevated serum levels of, for example, IL-1 $\beta$ in our study or IFN- $\alpha$ can diminish the HPA axis responsiveness as described above. ${ }^{21}$ In addition, longer-term elevated proinflammatory cytokines might influence steroidogenesis (was suggested, ref. 28). The question remains how and where steroidogenesis is disturbed.

Since mitochondria in adrenocortical cells are important organelles for steroidogenesis, ${ }^{16}$ they became a target of investigation in this study. It turned out that mitochondria are altered during the course of experimental arthritis characterised by swelling, rarefication of cristae/tubuli, more dispersed vesicles in and cavitations within the matrix. This picture resembles mitochondrial alterations when inhibitors of steroidogenesis such as aminoglutethimide and cyanoketosteroid are administered to rats. ${ }^{10} 29$ Aminoglutethimide inhibits the P450scc side chain cleavage enzyme and cyanoketosteroid inhibits the $3 \beta$-hydroxysteroid dehydrogenase; both enzymes can be blocked by TNF. ${ }^{1128}$ Thus, one might speculate that chronic presence of proinflammatory cytokines such as TNF or IL-1 $\beta$ might change mitochondrial morphology and function. Since the number of tissue density of intact mitochondria positively correlated with serum corticosterone levels, the presence of altered mitochondria can be causal for low glucocorticoid generation.

Another marker of normal mitochondria can be breakdown of lipid droplets in adrenocortical cells because cells with altered mitochondria might have decreased lipid droplet breakdown. The present study provided an electron microscopic morphology marker of lipid droplets, which can be separated in homogenous and inhomogenous. With the help of wild-type and ATGL-deficient mice, we were able to demonstrate that lipase-induced breakdown of lipid droplets is related to the presence of inhomogenous lipid droplets. From this point of view, inhomogenous droplets might be interpreted as activated lipid vacuoles that release cholesteryl ester into the cytoplasm, while homogenous lipid droplets might be interpreted as homogenous and quiescent fat vacuoles. Importantly, this study demonstrated that the density of inhomogenous lipid droplets correlated positively with density of normal mitochondria and negatively with density of altered mitochondria. In addition, the number of homogenous (inhomogenous) lipid droplets correlated negatively (positively) with plasma corticosterone in arthritic animals. Both facts support the concept that an impairment of mitochondria is related to altered lipid breakdown and, probably, steroidogenesis. These findings support the higher levels of Sudan III lipid staining in the overt phase of arthritic illness. 
In conclusion, inadequate secretion of glucocorticoids in relation to ongoing inflammation is visible in long-standing arthritis (inadequacy). IL-1 $\beta$ inhibits ACTH-stimulated secretion of glucocorticoids. It is accompanied by higher lipid storage in adrenocortical cells, altered mitochondrial morphology reminding to the influence of chemical steroidogenesis blockers and impaired lipid droplet breakdown. This study in experimental arthritis is the first to show marked adrenocortical morphological changes of critical organelles, which is paralleled by inadequate levels of corticosterone in chronic inflammation, and IL-1 $\beta$ seems to be key cytokine.

Acknowledgements We thank Heiko I Siegmund (central electron microscopy laboratory) for excellent technical assistance. We acknowledge the help of Dr Rudolf Zechner (Institute of Molecular Biosciences, University of Graz, Austria), who provided us with mice deficient for the gene of adipose triglyceride lipase (ATGL-deficient mice).

Contributors CW: development of the concept, generation of data, generating draft figures, drafting parts of the paper, final approval and generating the rebuttals. KK: generation of data, generating draft figures and final approval. JAS: providing study tools, revising the draft paper and final approval. RHS: development of the concept, drafting parts of the paper, generating final figures, final approval and generating the rebuttals.

Funding This study was supported by a grant of the Deutsche Forschungsgemeinschaft to RHS (STR 511/15-1,2,3 and FOR696 and STR 511/31-1).

\section{Competing interests None.}

Provenance and peer review Not commissioned; externally peer reviewed.

\section{REFERENCES}

1 Buttgereit F, Burmester GR, Straub RH, et al. Exogenous and endogenous glucocorticoids in rheumatic diseases. Arthritis Rheum 2011;63:1-9.

2 da Silva JA, Jacobs JW, Kirwan JR, et al. Safety of low dose glucocorticoid treatment in rheumatoid arthritis: published evidence and prospective trial data. Ann Rheum Dis 2006:65:285-93.

3 Howard RP, Venning EH, Fisk GH. Rheumatoid arthritis. II. Studies of adrenocortical and hypophyseal function and the effects thereon of testosterone and pregnenolone therapy. Can Med Assoc J 1950;63:340-2.

4 Cooper MS, Stewart PM. Corticosteroid insufficiency in acutely ill patients. N Engl J Med 2003;348:727-34.

5 Bornstein SR. Predisposing factors for adrenal insufficiency. N Engl I Med 2009;360:2328-39

6 Jessop DS, Harbuz MS. A defect in cortisol production in rheumatoid arthritis: why are we still looking? Rheumatology (Oxford) 2005:44:1097-100.

7 Straub R, Buttgereit F, Cutolo M. Alterations of the hypothalamic-pituitary-adrenal axis in systemic immune diseases-a role for misguided energy regulation. Clin Exp Rheumatol 2011;29:S15-23.

8 Straub RH, Paimela L, Peltomaa R, et al. Inadequately low serum levels of steroid hormones in relation to IL-6 and TNF in untreated patients with early rheumatoid arthritis and reactive arthritis. Arthritis Rheum 2002;46:654-62.
9 Del Rey A, Wolff C, Wildmann J, et al. Disrupted joint-immune-brain communication during experimental arthritis. Arthritis Rheum 2008;58:3090-9.

10 Kadioglu D, Harrison RG. The functional relationships of mitochondria in the rat adrenal cortex. J Anat 1971:110:283-96.

11 Herrmann M, Schölmerich J, Straub RH. Influence of cytokines and growth factors on distinct steroidogenic enzymes in vitro: a short tabular data collection. Ann N Y Acad Sci 2002;966:166-86.

12 Greenspan FS, Gardner DG. Basic and clinical endocrinology. New York: Lange MedicalBooks/McGraw-Hill, Medical Pub. Division, 2004.

13 Acton S, Rigotti A, Landschulz KT, et al. Identification of scavenger receptor SR-BI as a high density lipoprotein receptor. Science 1996;271:518-20.

14 Plump AS, Erickson SK, Weng W, et al. Apolipoprotein A-I is required for cholesteryl ester accumulation in steroidogenic cells and for normal adrenal steroid production. J Clin Invest 1996;97:2660-71.

15 Haemmerle G, Lass A, Zimmermann R, et al. Defective lipolysis and altered energy metabolism in mice lacking adipose triglyceride lipase. Science 2006;312:734-7.

16 Miller WL, Bose HS. Early steps in steroidogenesis: intracellular cholesterol trafficking. J Lipid Res 2011;52:2111-35.

17 Cutolo M, Foppiani L, Minuto F. Hypothalamic-pituitary-adrenal axis impairment in the pathogenesis of rheumatoid arthritis and polymyalgia rheumatica. J Endocrinol Invest 2002;25:19-23.

18 Koller MD, Templ E, Riedl M, et al. Pituitary function in patients with newly diagnosed untreated systemic lupus erythematosus. Ann Rheum Dis 2004;63:1677-80.

19 Imrich R, VIcek M, Aldag JC, et al. An endocrinologist's view on relative adrenocortical insufficiency in rheumatoid arthritis. Ann N Y Acad Sci 2010;1193:134-8.

20 Persellin RH, Kittinger GW, Kendall JW. Adrenal response to experimental arthritis in the rat. Am J Physiol 1972;222:1545-9.

21 Gisslinger $H$, Svoboda T, Clodi M, et al. Interferon-alpha stimulates the hypothalamic-pituitary-adrenal axis in vivo and in vitro. Neuroendocrinology 1993:57:489-95.

22 Benson RO, Anderson AW. Observations on the adrenal glands of normal swine and swine affected with arthritis. Am J Vet Res 1973;34:1247-51.

23 Straub RH, Besedovsky HO. Integrated evolutionary, immunological, and neuroendocrine framework for the pathogenesis of chronic disabling inflammatory diseases. FASEB J 2003;17:2176-83.

24 Bornstein SR, Engeland WC, Ehrhart-Bornstein M, et al. Dissociation of ACTH and glucocorticoids. Trends Endocrinol Metab 2008;19:175-80.

25 Larsen $C M$, Faulenbach M, Vaag A, et al. Interleukin-1-receptor antagonist in type 2 diabetes mellitus. N Engl I Med 2007;356:1517-26.

26 Straub RH, Cutolo M, Buttgereit F, et al. Energy regulation and neuroendocrine-immune control in chronic inflammatory diseases. J Intern Med 2010;267:543-60.

27 Straub RH. Evolutionary medicine and chronic inflammatory state - known and new concepts in pathophysiology. J Mol Med 2012;90:523-34.

28 Jäättelä $M$, Ilvesmaki V, Voutilainen $R$, et al. Tumor necrosis factor as a potent inhibitor of adrenocorticotropin- induced cortisol production and steroidogenic P450 enzyme gene expression in cultured human fetal adrenal cells. Endocrinology 1991;128:623-9.

29 Finegold MJ, Greene LE. Mitochondrial damage in experimental congenital adrenal hyperplasia. J Cell Biol 1970;45:455-61. 
ARD Inadequate corticosterone levels relative to arthritic inflammation are accompanied by altered mitochondria/cholesterol breakdown in adrenal cortex: a steroid-inhibiting role of $\mathrm{IL}-1 \beta$ in rats

Christine Wolff, Katharina Krinner, Josef A Schroeder and Rainer $\mathrm{H}$ Straub

Ann Rheum Dis 2015 74: 1890-1897 originally published online May 7, 2014

doi: 10.1136/annrheumdis-2013-203885

Updated information and services can be found at:

http://ard.bmj.com/content/74/10/1890

These include:

Supplementary Supplementary material can be found at:

Material http://ard.bmj.com/content/suppl/2014/05/08/annrheumdis-2013-2038 85.DC1

References This article cites 28 articles, 7 of which you can access for free at: http://ard.bmj.com/content/74/10/1890\#BIBL

Email alerting Receive free email alerts when new articles cite this article. Sign up in the service box at the top right corner of the online article.

Topic Articles on similar topics can be found in the following collections

Collections

Degenerative joint disease (4641)

Musculoskeletal syndromes (4951)

Immunology (including allergy) (5144)

Inflammation (1251)

Connective tissue disease (4253)

Rheumatoid arthritis (3258)

\section{Notes}

To request permissions go to:

http://group.bmj.com/group/rights-licensing/permissions

To order reprints go to:

http://journals.bmj.com/cgi/reprintform

To subscribe to BMJ go to:

http://group.bmj.com/subscribe/ 